\title{
BRAIN STRUCTURE LOCALIZATION IN POSITRON EMISSION TOMOGRAPHY: COMPARISON OF MAGNETIC RESONANCE IMAGING AND A STEREOTACTIC METHOD
}

\author{
James M. Mountz ${ }^{1}$ and Mark W. Wilson ${ }^{2}$ \\ Division of Nuclear Medicine ${ }^{1}$, Department of Internal Medicine, University of Michigan Medical Center, \\ Ann Arbor, MI and The Henry Ford Hospital ${ }^{2}$, Department of Intemal Medicine, Detroit, MI
}

\author{
(Received 6 March 1990; Revised 17 August 1990)
}

\begin{abstract}
A method commonly used for localization of brain regions on positron emission tomographic (PET) images is direct visualization and designation of structure on the image itself. This technique, however, is limited to portions of the brain having sufficient differential radionuclide uptake to permit the recognition of structure by observers familiar with brain anatomy. Two other methods commonly used instead of direct visualization are magnetic resonance imaging (MRI) and stereotactic methods of localization. This report compares the accuracy of a MRI method versus a stereotactic method of brain structure localization on PET. The three localization methods were analyzed for sixteen different brain regions in 5 different subjects by two independent observers. The results were converted to $P E T$ pixel size $(1$ pixel $=.127 \mathrm{~cm})$ for comparison. MRI localization differed from direct visualization by a mean and standard deviation of $\sim 2 \pm 1$ pixels in both the $X$ and $Y$ dimensions. Stereotactic localization differed from direct visualization by $-2 \pm 1$ pixels in the $X$ dimension and by $\sim 6 \pm 2$ pixels in the $Y$ dimension. This larger variation seen with the stereotactic method may be attributed to the questionable assumption of linearity of structure location with respect to size of the inner table of the calvarium.
\end{abstract}

Key Words: Brain, PET, MRI, Stereotaxis, Neuroanatomic localization

\section{INTRODUCTION}

Anatomic localization techniques assist in the analysis of functional cerebral image data (1-5). Methods to localize brain structure on Positron Emission Tomography (PET) are becoming more important as the applications of PET broaden $(6,7)$. Direct visual identification of brain structure on PET images eliminates the added time and cost of obtaining anatomic radiographic studies which are required for the employment of comparative techniques such as MRI or stereotactic methods. Direct visualization and identification of brain anatomy on PET images is generally conducive for structure identification only when tracer uptake is significantly different between adjacent brain tissues, and its utility in PET studies with more specfic regional tracer uptake (e.g., receptor binding studies) is limited (8). MRI localization $(9,10)$ bestows excellent anatomic resolution, but requires additional costs and application of methods to insure reproducibility of patient orientation and slice alignment between scanners. Stereotactic methods (11, 12) are useful, but one of the more commonly used

Correspondence and reprint requests: James M. Mountz, M.D. Ph.D., Associate Professor of Radiology, University of Alabama Medical Center, 619 19th Street, Birmingham, AL 35233. stereotactic techniques (12) requires the acquisition of a lateral skull radiograph and determination of stereotactic parameters, as well as the development and utilization of computer software. In addition, this stereotactic method assumes a linear transformation relationship exists between brain structures and the size of the inner table of the calvarium, and accurate use assumes subjects have normal brain anatomy. The use of this method cannot correct for normal anatomic variation of brain anatomy.

We undertook a study to test the precision of brain structure localization by comparing the relative accuracy of these two commonly employed techniques. By using ${ }^{15} \mathrm{O}-\mathrm{H}_{2} \mathrm{O}$ PET scans we defined 16 unequivocally identifiable brain structures and compared their PET scan location with accurately aligned MRI scans, and a commonly used stereotactic method (12).

\section{MATERIALS AND METHODS}

The study population consisted of 5 female subjects, aged 22-44, with normal brain structure as determined by MRI. Prior to each study each subject signed a consent form approved by the human studies review board at The University of Michigan. All PET scans were performed in the Division of Nuclear Medicine at The University of Michigan Medical Center. PET scans 
were performed on a TCC PC-4600 tomograph having in-plane resolution of $10.5 \mathrm{~mm}$ full width half maximum (FWHM), with axial resolution of $10 \mathrm{~mm}$ FWHM, and an interslice distance of $11 \mathrm{~mm}$. The pixel dimensions $(128 \times 128)$, sensitivity, and operating characteristics have been previously described (13). Each morning, prior to scanning, the PET scanner was calibrated to normalize detector response across all detectors. Each subject was scanned in a resting state following an intravenous injection of approximately $35 \mathrm{mCi}$ of ${ }^{15} \mathrm{O}$ $\mathrm{H}_{2} \mathrm{O}$ blood flow tracer. A six minute dynamic sequence of 9 scans $(6 \times 20 \mathrm{secs} ; 2 \times 60 \mathrm{secs} ; 1 \times 120 \mathrm{sec})$ was acquired beginning at the time of injection. Continuous blood sampling from the left radial artery permitted calculation of the radiotracer input function to brain. A weighted integral estimation approach of Alpert et al. (14) modified as described by Koeppe et al. (15) was used for calculation of cerebral blood flow yielding regional values in $\mathrm{mL} / 100 \mathrm{~g}$-min. Each scan produced images from five planes oriented parallel to the canthomeatal line from an inferior level approximately bisecting the gyrus rectus anterior and the mid-cerebellum posterior to a superior level approximately transecting the supracallosal portion of the cingulum.

The MRI images were acquired using the Diasonics MT/S superconducting system operating at 0.35 Tesla. The operating characteristics of this imager have been previously described in detail (16). Quality control of the MR scanner was performed prior to imaging using a distortion test phantom constructed of 60 parallel square rods constructed of plexiglas surrounded by a solution of manganese chloride (17). The average anisotrophy over a large body size was approximately 2 to $3 \%$ (18). Images of contiguous $10 \mathrm{~mm}$ axial sections were obtained in all patients utilizing pulse sequences of repetition time $0.5,1.0 \mathrm{sec}$, and echo delay times of $28,56 \mathrm{~m}$ $\mathrm{sec}$. Images were reconstructed using two-dimensional Fourier transformation of a $256 \times 256$ element matrix grid. Voxel sizes were $1.7 \times 1.7 \times 5.0 \mathrm{~mm}$. The $\mathrm{T}_{2}$ weighted images were used for comparison with PET because of the greater grey to white matter anatomic distinction. The third of five MR planes was positioned through the center of the third of five PET planes using laser localization along the identical line used during PET scanning. Registration of image planes was performed after careful laser beam alignment of external reproducible landmarks $(\mathrm{OM}$ line $+1.0 \mathrm{~cm})$ defined as the center plane with two adjacent planes above and below this reference line. Since the PET interslice $\Delta Z=$ $11.0 \mathrm{~mm}$ and the MRI interslice $\Delta Z=10.0 \mathrm{~mm}$ this method limited axial misregistration between MRI and PET slices to $1.5 \mathrm{~mm}$ at the extreme (first and fifth) planes. Patient motion during each scan was limited to 2 $\mathrm{mm}$, as confirmed by checking the laser line position before and after the scan with respect to the bony lateral canthus and the external auditory meatus which have been shown to be reproducible external landmarks for positioning (10). All MR images were compared with PET images after scaling the MRI pixel size to match the PET pixel size. This was performed by measuring the maximum longitudinal $(Y)$ and horizontal $(X)$ size of the brain (in pixels) on the MR and PET image. A common origin $(0,0)$ was defined at the lower left of the PET image, and the MR image. The MR image was scaled such that the most anterior and right side of the brain had the same coordinates as the corresponding PET image. Each structure coordinate location $(X, Y)$ was displayed on the image consol after a curser was placed over the center of the structure. This procedure was performed separately for each of the 5 sections on all subjects. No attempt was made to use external fiduciary markers or other more sophisticated matching techniques $(4,10)$ since the intent of this study was to compare the more widely utilized and commonly employed localization methods.

The stereotactic method was performed after obtaining a lateral skull $\mathrm{X}$-ray, determining the locations of the anterior commissure, posterior commissure (AC-PC), and glabella-inion (GI) lines. The other stereotactic parameters necessary to perform the translation between the stereotactic coordinate system and the PET coordinate system were entered into a program which localized each of the 16 structures (12).

Sixteen brain regions on each patient were identified both by MRI and the stereotactic technique, and the coordinates were compared with the coordinate values obtained by direct visualization of definitely identifiable structures on the ${ }^{15} \mathrm{O}-\mathrm{H}_{2} \mathrm{O}$ PET images (regions selected by two physicians with knowledge of gross brain anatomy). Differences (in pixels) were determined for MRI and stereotaxis compared with PET to determine the relative accuracy of each method to identify brain structure. Regions which could be unequivocally identified using all three methods were: the carotid arteries at the level of the carotid siphon bilaterally, cavernous sinuses bilaterally, center of brain stem at the level of the top of the petrous pyramids, center of the primary visual cortex, heads of the right and left caudate nuclei, and position at edge of brain on the MR scan identified by extension of a horizontal line passing through each respective structure, genue of the internal capsule bilaterally, and right and left thalami and position at edge of brain on the MR scan identified by extension of a horizontal line passing through each respective structure. The location of pixel and plane position on the PET images of the 16 regions was performed without reference to the MRI scan. The transverse PET coordinates were defined by pixel location $(X, Y)$ for each of 
Table 1. Average difference between coordinate position localized by PET, MRI, and the stereotactic method for $X$, $Y$, and $Z$ for the 5 subjects (difference \pm SEM). $\Delta \mathrm{MRI}=$ [PET location - MRI location], and $\Delta$ Stereo $=[$ PET location - Stereotactic location]. Please see Fig. 1 legend for structure abbreviations.

\begin{tabular}{|c|c|c|c|c|c|c|}
\hline Structure & $\Delta \mathbf{X R I}$ & $\Delta \mathbf{Y M R}$ & $\Delta \mathbf{M R}$ & $\Delta \mathbf{M}$ Stereo & $\Delta$ Y Stereo & $\Delta \mathbf{Z}$ Stereo \\
\hline RG & $1.4 \pm 0.80$ & $-2.25 \pm 0.67$ & $0.78 \pm 2.59$ & $-0.2 \pm 1.16$ & $0.60 \pm 0.51$ & $-0.94 \pm 1.49$ \\
\hline LG & $-1.0 \pm 0.72$ & $-1.20 \pm 0.67$ & $0.78 \pm 2.59$ & $0.6 \pm 0.51$ & $0.80 \pm 0.58$ & $-0.94 \pm 1.49$ \\
\hline PVC & $-0.2 \pm 1.07$ & $0.20 \pm 0.72$ & $1.56 \pm 1.57$ & $0.6 \pm 0.67$ & $0.20 \pm 0.73$ & $-1.40 \pm 0.90$ \\
\hline LC & $-1.8 \pm 0.58$ & $-1.20 \pm 0.58$ & $0.78 \pm 2.59$ & $-1.4 \pm 1.03$ & $0.80 \pm 0.38$ & $1.25 \pm 1.75$ \\
\hline RC & $0.0 \pm 0.63$ & $-0.80 \pm 1.07$ & $0.78 \pm 2.59$ & $-0.2 \pm 0.58$ & $1.60 \pm 0.25$ & $1.25 \pm 1.75$ \\
\hline LCBE & $-1.6 \pm 0.94$ & $-0.40 \pm 1.03$ & $0.78 \pm 2.59$ & $0.0 \pm 1.74$ & $2.00 \pm 0.45$ & $1.25 \pm 1.75$ \\
\hline RCBE & $0.2 \pm 0.67$ & $-0.80 \pm 1.16$ & $0.78 \pm 2.59$ & $-1.0 \pm 1.05$ & $2.00 \pm 0.32$ & $1.25 \pm 1.75$ \\
\hline LCS & $0.2 \pm 0.49$ & $0.20 \pm 0.80$ & $1.56 \pm 1.57$ & $-0.2 \pm 0.58$ & $3.00 \pm 1.82$ & $0.47 \pm 1.03$ \\
\hline RCS & $0.4 \pm 0.49$ & $0.80 \pm 0.67$ & $1.56 \pm 1.57$ & $2.2 \pm 0.73$ & $3.00 \pm 2.08$ & $0.47 \pm 1.03$ \\
\hline LT & $0.4 \pm 1.03$ & $0.20 \pm 0.72$ & $-1.56 \pm 1.57$ & $0.8 \pm 1.03$ & $3.60 \pm 0.80$ & $-2.34 \pm 1.90$ \\
\hline LTBE & $0.0 \pm 1.21$ & $0.20 \pm 0.72$ & $-1.56 \pm 1.57$ & $-0.2 \pm 1.57$ & $3.80 \pm 0.80$ & $-2.34 \pm 1.90$ \\
\hline RTBE & $-0.8 \pm 0.49$ & $0.00 \pm 0.85$ & $-1.56 \pm 1.57$ & $-1.2 \pm 0.94$ & $3.80 \pm 0.80$ & $-2.34 \pm 1.90$ \\
\hline RT & $-1.0 \pm 0.31$ & $0.00 \pm 1.21$ & $-1.56 \pm 1.57$ & $-1.8 \pm 0.49$ & $3.80 \pm 0.80$ & $-2.34 \pm 1.90$ \\
\hline BS & $0.0 \pm 0.45$ & $-1.20 \pm 1.12$ & $-1.56 \pm 1.57$ & $0.0 \pm 0.45$ & $5.80 \pm 1.16$ & $-0.16 \pm 1.70$ \\
\hline LCA & $1.8 \pm 0.85$ & $1.40 \pm 1.88$ & $1.56 \pm 1.57$ & $0.0 \pm 1.21$ & $5.60 \pm 1.39$ & $-3.12 \pm 2.20$ \\
\hline RCA & $-1.0 \pm 0.54$ & $0.40 \pm 1.30$ & $1.56 \pm 1.57$ & $-0.2 \pm 0.67$ & $5.80 \pm 1.39$ & $-3.12 \pm 2.20$ \\
\hline
\end{tabular}

the structures. The location of structure in the $Z$ dimension was determined to the nearest plane on which it appeared on PET or MRI since the inherent interslicc distance did not allow more accurate placement. The stereotactic coordinates were given in planes and fractions of planes, and therefore structure location (in the $Z$ dimension) was placed in the nearest plane to permit comparison with PET and MRI. $Z$ coordinate values were converted to pixel location values for comparison purposes.

Each of the three methods of localization were validated by the two independent observers who localized each of the 16 structures using each method. The observers were instructed to place the cursor at the point of highest (or lowest) tracer uptake in each region of interest on the PET images, or identify what appeared to be the corresponding geometric center of the region on the MRI and stereotactic atlas sections.

MRI localization was performed by localizing each structure on the MR image (independent of the PET image) and comparing the MRI to PET pixel coordinates with those obtained by direct visualization of the PET image. Stereotactic localization was tested by localizing each structure from the anatomic atlas (19) and comparing the results of PET pixel coordinates obtained using the stereotactic method. This was also performed independently of the PET or MRI image.

Comparison of the accuracy of the MRI method versus the stereotactic method was performed by subtracting the location for the $X, Y$, and $Z$ coordinate for all 16 structures for each patient from the location found on the PET image. A total difference $\Delta r=\left(\Sigma^{5}{ }_{i=1}\right.$ $\left.\left(\Delta X_{i}^{2}+\Delta Y_{i}^{2}+\Delta Z_{i}^{2}\right)^{1 / 2} / 5\right)$ (since all structures were observed once in each of the 5 subjects) was then calculated to represent the 3 dimensional spatial distance from the PET coordinate.

To verify proper utilization of the stereotactic method at our institution, replication of the validation of this method was performed in an identical manner as presented by other investigators (12) by localization of the right and left cavernous sinuses. The cavernous sinuses are visualized as regions of decreased tracer uptake on the 6 minute sequence ${ }^{15} \mathrm{O}-\mathrm{H}_{2} \mathrm{O}$ PET scan since the average venous activity will drop below brain tissue activity after approximately one minute. This will result in the region being identified as having lower counts compared to surrounding brain tissue (15).

\section{RESULTS}

To validate the accurate employment of the stereotactic method at our institution, the identical structures (right and left cavernous sinuses) found by other investigators were localized. The right cavernous sinus had mean stereotactic localization coordinates of $X=.83 \pm$ $.18 \mathrm{~cm}, Y=1.22 \pm .47 \mathrm{~cm}$ and $Z=-2.0 \pm .32 \mathrm{~cm}$, and likewise the localization of the left cavernous sinus had mean stereotactic coordinates of $X=-.79 \pm .19$ $\mathrm{cm}, Y=1.32 \pm .47 \mathrm{~cm}$, and $Z=-2.10 \pm 3.7 \mathrm{~cm}$. All values were within one standard deviation of the values found by other investigators using the stereotactic method (12). Inter-observer agreement on structure coordinates identified on PET and MRI agreed for all 16 regions between the two independent observers in the $X$ and $Y$ dimensions to within 1 pixel and to the same plane in the $Z$ dimension for all structures localized.

Table 1 gives the results of comparing PET to MRI and PET to the stereotactic method for each dimension $X, Y$, and $Z$. Figure 1 shows the comparison between the stereotactic method and MRI in localizing the 16 structures identified on the PET image. Structures best localized were: the genue of the internal capsule, the primary visual cortex, and the heads of the caudate 


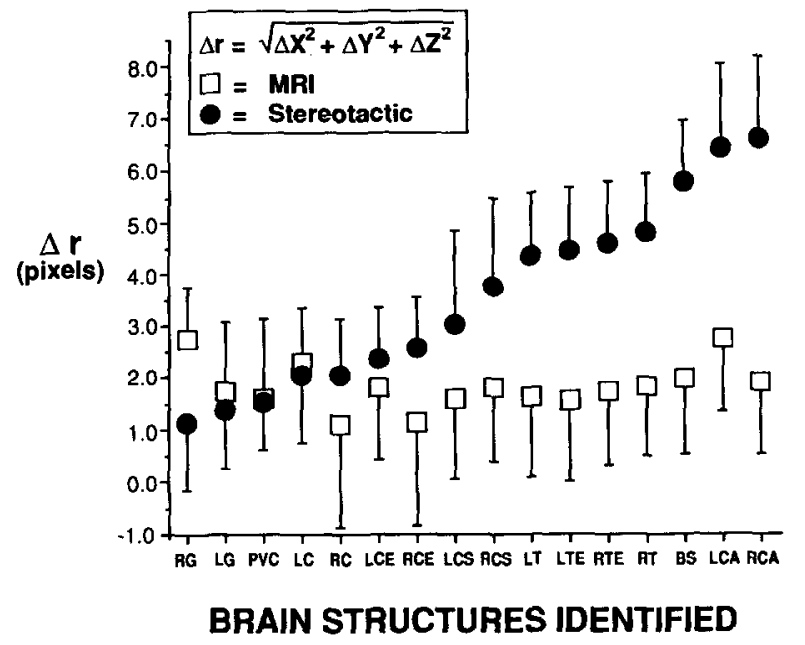

Fig. 1. Graphically shown is the comparison between the MRI and the stereotactic method of localization for the sixteen structures identified on the PET scan. The difference in agreement between the two methods is given by $\Delta r$, as previously defined. The MRI method of localization yielded a difference, on average, of 2 pixels, while the stereotactic method yielded a difference as large as 6 pixels. Data are expressed as differences \pm SEM. Abbreviations: $R G=$ genue at right internal capsule, LG $=$ genue of left internal capsule, PVC $=$ primary visual cortex, $\mathrm{LC}=$ left caudate, $\mathrm{RC}=$ right caudate, $\mathrm{LCBE}=$ brain edge adjacent to left caudate, RCBE = brain edge adjacent to right caudate, LCS $=$ left cavernous sinus, $\mathrm{RCS}=$ right cavernous sinus, $\mathrm{LT}=$ left thalamus, LTBE = brain edge adjacent to left thalamus, $\mathrm{RTBE}=$ brain edge adjacent to right thalamus, $\mathrm{RT}=$ right thalamus, $\mathrm{BS}=$ brain stem, LCA $=$ left carotid artery siphon, RCA = right carotid artery siphon.

nucleus. For these structures agreement was within approximately 2 pixels $(1$ pixel $=0.127 \mathrm{~cm})$. However, for the thalami, brain stem, and carotid artery siphon, agreement was less accurate for the stereotactic method as compared to the MRI method. Figure 2 exemplifies our findings in three representative structures: head of the left caudate nucleus, right internal carotid artery siphon, and brain stem. There is relatively good location of the head of the left caudate nucleus for both the MRI and stereotactic method as compared to the visualized structure on the PET image; however, it is noted that identification on MRI agrees better than the stereotactic method for the right internal carotid artery siphon and brain stem.

\section{DISCUSSION}

Structure identification is critical for interpretation of PET images, and the accuracy of the method of anatomic localization is becoming increasingly important with higher resolution PET scanners. In this investiga-
January-February/1991, Volume 15, Number 1

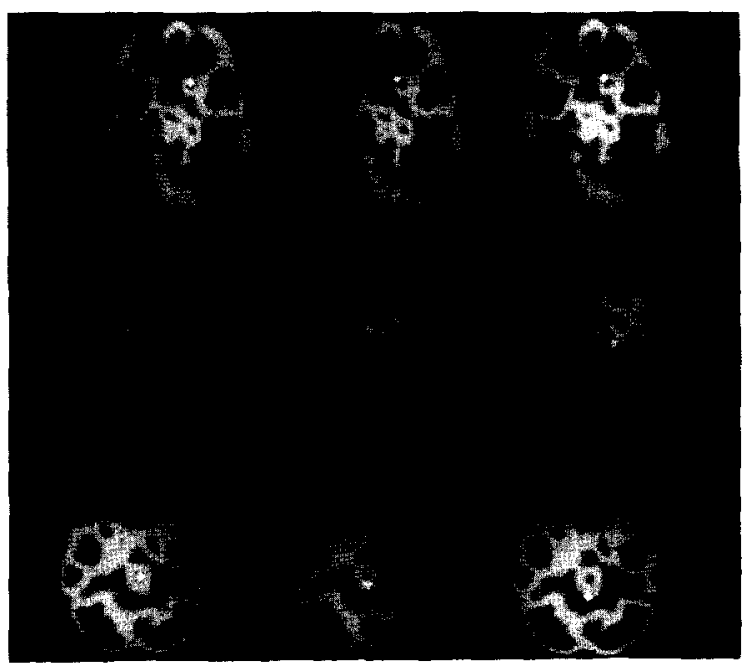

Fig. 2. Examples of the accuracy of direct visualization, MRI, and stereotaxis respectively for 3 representative structures (left caudate - TOP, right carotid artery siphon - MIDDLE, and brain stem - BOTTOM). The first column of images depicts the cursor location as determined by the independent observers on the PET image via direct visualization. The following images show that there is more accurate localization using MRI identification of these 3 structures (second column) as compared to stereotactic localization of these 3 structures (third column).

tion three commonly employed localization methods were investigated. The PET image was selected as the reference standard since it contains the structure for desired identification. No correction for partial volume effects, or slight anatomic misregistration between MRI and PET was used, since these methodologies are not generally available. Of the three methods of structure localization presented, good correlation was observed for all structures using MRI identification, however, a greater variation in $\Delta r$ values was observed using the stereotactic method of localization compared with MRI for structures closer to the base of the brain. The results demonstrate that the use of commonly available MRI localization techniques (requiring only reproducible head positioning using laser alignment) are more accurate than the stereotactic method. Presumable the employment of MRI registration (4) or coordinated transformation techniques (10) would result in greater accuracy in MRI localization. However, further investigations employing these more sophisticated MRI localization methods will be necessary to verify this. The $X$ coordinate agreed best for both methods, however this is expected since the stereotactic method uses the PET image to determine the lateral ( $X$ dimension) of brain width. Errors in the stereotactic method may be related to the assumption that brain structures translate in a linear manner as calvarial sizes differ. It is of note 
that the greatest inaccuracy in the stereotactic method occurred along the $Y$ dimension (an in-plane coordinate which was reliably measured in our study). This suggests that the error in the stereotactic method may be associated with the high prevalence of variance in longitudinal cranial dimensions in the normal population (20) and nonlinear movement of brain structures in this dimension.

The MRI method of localization, in contrast, is patient-specific, allowing for individual anatomic variation between patients; and therefore is free of error in measurements from a skull radiograph. In addition, it makes no assumptions as to the linearity of the differences in locations of brain structure with respect to the geometry of the calvarium. However, one potential source of inaccuracy of comparing MRI with PET is the assumption that the highest (or lowest) region of tracer uptake on ${ }^{15} \mathrm{O}-\mathrm{H}_{2} \mathrm{O}$ PET corresponds to the center of the corresponding structure of the MRI or the stereotactic atlas section. In conclusion, we found that anatomic localization of brain structure using a commonly employed MRI method, is more accurate than the stereotactic method. Our results suggest that the use of MRI methods after correction for partial volume effects, magnetic field inhomogeneity (21), anatomic slice and position mis-registration (4), and coordinated system alignment correction (10) should provide a method for more accurate localization of structure on PET images.

\section{SUMMARY}

The objective of this investigation was to compare three commonly used methods for localization of structure on functional positron emission tomography (PET) images: namely, direct visual identification of structure on the PET image compared with magnetic resonance imaging (MRI) and a stereotactic technique. Two independent observers were employed to define structure and to verify that inter-observer variability was minimal. MRI more accurately and consistently identified structures as compared to the stereotactic method, regardless of their location in the brain. The $x$ coordinate agreed best for both methods, however this was expected since the stereotactic method uses the PET image to determine the lateral ( $x$ dimension) of brain width. It is of note that the greatest inaccuracy of the stereotactic method occurred along the $y$ dimension (an in plane coordinate which was reliably measured in our study). This supports the notion that errors in the stereotactic method may be associated with the high prevalence of variance in longitudinal cranial dimensions in the normal population and the non-linear motion of brain structures in this dimension. The MRI method of localization, in contrast, is patient-specific, allowing for individual anatomic variation between patients; and therefore is free of error in measurements from a skull radiograph. In addition, it makes no linearity assumption regarding differences in locations of brain structure with respect to the geometry of the calvarium as does the stereotactic method. In conclusion, we found that anatomic localization of brain structure employing MRI is more accurate than the stereotactic method.

\section{REFERENCES}

1. Conti, J.; Deck, M.D.F.; Rottenberg, D.A. An inexpensive video patient respositioning system for use with transmission and emission computed tomographs. J. Comput. Assist. Tomogr. 6(2): 417-421; 1982.

2. Herholz, K.; Pawlik, G.; Wienhard, K.; Heiss, W-D. Computer assisted mapping in quantitative analysis of cerebral positron emission tomograms. J. Comput. Assist. Tomogr. 9(1):154-161; 1985.

3. Levin, D.N.; Hu, X.: Tan, K.K., et al. The brain: Integrated three-dimensional display of MR and PET images. Radiology 172: 783-789; 1989.

4. Pelizzari, C.A.; Chen, G.T.Y.; Spelbring, D.R.; Weichselbaum, R.R.; Chen, C-T. Accurate three-dimensional registration of CT, PET, and/or MR images of the brain. J. Comput. Assist. Tomogr. 13(1):20-26; 1989.

5. Perlmutter, J.S.; Herscovitch, P.; Powers, W.J.; Fox, P.T.; Raichle, M.E. Standardized mean regional method for calculating global positron emission tomographic measurements. J. Cereb. Blood Flow Metab. 5:476-480; 1985.

6. Adair, T.; Karp, P.; Stein, A.; Bajcsy, R.; Reivich, M. Computer assisted analysis of tomographic images of the brain. J. Comput. Assist. Tomogr. 5(6):929-932; 1981.

7. Levin, D.N.; Pelizzari, C.A.; Chen, G.T.Y.; Chen, C-T.; Cooper, M.D. Retrospective geometric correlation of MR, CT, and PET images. Radiology 169:817-823; 1988.

8. Sedvall, G.: Farde, L.: Persson, A.: Weisel, F-A. Imaging of neurotransmitter receptors in the living human brain. Arch. Gen. Psychiatry 43:995-1005; 1986.

9. Evans, A.C.; Beil, C.; Marrett, S.; Thompson, C.J.; Hakim, A. Anatomical-functional correlation using an adjustable MRI-based region of interest atlas with positron emission tomography. J. Cercb. Blood Flow Metab. 8:513-530; 1988.

10. Wilson, M.W.; Mountz, J.M. A reference system for neuroanatomical localization on functional reconstructed cerebral images. J. Comput. Assist. Tomogr. 13(1):174-178; 1989.

11. Bohm, C.; Greitz, T.; Kingsley, D.; Berggren, B.M.; Olsson, L. Adjustable computerized stereotaxic brain atlas for transmission and emission tomography. AJNR 4:731-733; 1983.

12. Fox, P.T.; Perlmutter, J.S.; Raichle, M.E. A stereotactic method of anatomical localization for positron emission tomography. J. Comput. Assist. Tomogr. 9(1):141-153; 1985.

13. Kearfott, K.J.; Carroll, L.R. Evaluation of the performance characteristics of the $\mathrm{PC} 4600$ positron emission tomograph. J. J. Comput. Assist. Tomogr. 8(3):502-513; 1984.

14. Alpert, N.M.; Eriksson, L.; Chang, J.Y.; Bergstrom, M.; Litton, J.E.; Correia, J.A.; Bohm, C.; Ackerman, R.H.; Taveras, J.M. Strategy for the measurement of regional cerebral blood flow using short-lives tracers and emission tomography. J. Cereb. Blood Flow Metabol. 4:28-34; 1984.

15. Koeppe, R.A.; Hutchins, G.D.; Rothley, J.M.; Hichwa, R.D. Examination of assumptions for local cerebral blood flow studies in PET. J. Nucl. Med. 28:1695-1703; 1987. 
16. Crooks, L.; Arakawa, M.; Hoenninger, J.; et al. Nuclear magnetic resonance whole-body imager operating at 3.5 KGauss. Radiology 143:169-174; 1982 .

17. Covell, M.M.; Hearshen, D.O.; Carson, P.L.; Chenevert, T.P.; Shreve, P.; Aisen, A.M.; Bookstein, F.L.; Murphy, B.W. Automated analysis of multiple performance characteristics in magnetic resonance imaging systems. Med. Phys. 13:815-823; 1986.

18. Carson, P.L.; Ellis, J.; Murphy, B.W.; Zhang, Y.; Liu, G. Automated analysis of magnetic resonance imaging system performance. In: Dixon, R.L., ed. Acceptance testing and quality control. In The Proceedings of the AAPM Symposium. PP 151-173. April 6-8, 1988.

19. Talairach, J.; Szikla, G.; Tournoux, P.; Prossalentis, A.; BordasFerrer, M.; Covello, L.; Iacob, M.; Mempel, E. Atlas of stereotaxic anatomy of the telencephalon. Masson \& $\mathrm{C}^{\mathrm{ie}}$, Editeurs, Saint-Germain, Paris; 1967.

20. Lewin, T.; Skrobak-Kaczynski, J.; Sigholm, G. Secular changes in craniofacial dimensions in a homogenous population. Acta Morphol. Neerl-Scand. 11:289-319; 1973:

21. Bellon, E.M.; Haacke, E.M.; Coleman, P.E.; Sacco, D.C.; Steiger, D.A.; Gangarosa, R.E. MR artifacts: A review. AJR $147: 1271-1281 ; 1986$.

About the Author-James Michael MounTz received his Ph.D. in Physics from Michigan State University in 1975. After completing two years as a National Science Foundation postdoctoral fellow, he entered medical school at Case Western Reserve University and received his M.D. in 1981. Dr. Mountz performed his internship and residency in Radiology and fellowship in Nuclear Medicine at the University of Michigan. He became a Diplomat of the American Board of Radiology in 1986, and a Diplomat of the American Board of Nuclear Medicine in 1987. He joined the staff of the Department of Internal Medicine, Division of Nuclear Medicine, at the University of Michigan where he is now an Assistant Professor. His work has primarily focused on functional imaging, and he has written numerous papers on quantitative and localization techniques. Dr. Mountz is a member of the Sigma Pi Sigma physics honor society and the Phi Eta Tau scholastic honor society. He and Dr. Wilson have similar scientific interests, and together they have received a U.S. patent for the invention of system and method for determining orientation of planes of imaging.

About the Author-Mark WILLIAM WILSON received his B.S. degree in Chemical Engineering from the University of Michigan in 1986 , and his M.D. degree from the University of Michigan in 1990. Dr. Wilson is appointed to enter the residency program in Radiology at the University of California-San Francisco in 1991 after completion of his internship at Henry Ford Hospital in Detroit. Dr. Wilson has used his engineering and medical backgrounds to pursue and solve problems in radiology and nuclear medicine quantification, and functional imaging localization methods. He has also developed methods to measure in vitro vascular reactivity using an intravascular ultrasound catheter. Dr. Wilson is a member of Tau Beta Pi engineering honor society. 